\section{Preoperative CT scans might overestimate the size of renal tumors}

The size of renal tumors on CT or X-ray images is often used to decide whether a patient is suitable to undergo nephron-sparing surgery (NSS). Previous studies that compared the apparent size of renal tumors on CT or X-ray images with the actual size of pathologic specimens have yielded conflicting results. Schlomer and colleagues, therefore, conducted a retrospective study in 126 patients with a renal lesion suspicious for renal cell carcinoma, in whom CT was performed $\leq 60$ days before surgery. Clinical size was defined as the largest tumor measurement on CT; pathologic size was the largest measurement seen at pathology.

For all 133 tumors detected, mean clinical size did not differ significantly from mean pathologic size. In terms of tumor stage, clinical size was markedly greater than pathologic size only for tumors $>4 \mathrm{~cm}$ and graded T1a or T3a. Mean clinical size was larger than pathologic size for tumors $>7 \mathrm{~cm}$; this difference was substantial for tumors $1-2,2-3,3-4$, and $4-5 \mathrm{~cm}$ in size.

The greatest difference between mean clinical and mean pathologic size $(0.87 \mathrm{~cm})$ was seen for tumors $4-5 \mathrm{~cm}$ in size, which the authors suggest might lead to some patients with tumors of clinical size $<4 \mathrm{~cm}-$ who are usually suitable for NSS-not receiving this treatment; additionally some patients with tumors of clinical size $>4 \mathrm{~cm}$ might be suitable for NSS after consultation. Prospective studies that examine other factors associated with the difference between clinical and pathologic size (including tumor location and presence of hematomas) are required.

Original article Schlomer B et al. (2006) How does the radiographic size of a renal mass compare with the pathologic size? Urology 68: 292-295

\section{Antimuscarinic agents might impair memory in older people}

A joint Swiss and US team has found that some antimuscarinic agents could have deleterious effects on memory in older, healthy adults.

In this multicenter, randomized, double-blind study, 150 healthy men and women (aged $>60$ years) were randomly allocated to 3 weeks of treatment with either placebo, darifenacin (7.5 mg in weeks 1 and 2; $15 \mathrm{mg}$ in week 3 ), or extended-release oxybutynin (10 mg in week 1 , $15 \mathrm{mg}$ in week 2, $20 \mathrm{mg}$ in week 3). Dose escalation allowed steady-state cerebrospinal fluid concentrations to be reached. Cognitive function tests were performed at weeks $0,1,2$, and 3 . Patients self-reported any subjective memory changes, and were evaluated for accuracy of recall in the Name-Face Association Test.

Patients given oxybutynin showed markedly less accuracy in delayed recall on the NameFace Association Test at week 3, compared with those given darifenacin or placebo-a deficit comparable to that caused by 10 years of 'normal' aging. Darifenacin was not associated with any significant memory changes, and no differences were seen between groups in terms of subjective memory changes.

M1 muscarinic receptors are important in memory and cognition, which might help to explain the differential effects of oxybutynin (which binds to $\mathrm{M} 1$ and $\mathrm{M} 3$ receptors) and darifenacin (which binds selectively to $\mathrm{M} 3$ receptors) on memory. The effects of extended-release oxybutynin should now be explored in a setting that is more representative of clinical practicenamely, in patients who have pre-existing cognitive impairment, and in those who are receiving other anticholinergic medications.

Original article Kay G et al. (2006) Differential effects of the antimuscarinic agents darifenacin and oxybutynin ER on memory in older subjects. Eur Urol 50: 317-326

\section{Toremifene could prevent prostate cancer in high-risk patients}

The selective estrogen-receptor (ER) modulator toremifene can either stimulate or block ERs in target tissues. In the prostate, toremifene blocks ERs, and is under evaluation as a chemopreventive treatment in men with highgrade prostatic intraepithelial neoplasia, who are at high risk of developing prostate cancer.

Price and colleagues' double-blinded, placebo-controlled, dose-finding study included 514 men with biopsy-confirmed high-grade prostatic intraepithelial neoplasia but without initial evidence of prostate cancer. The patients were randomly allocated to daily treatment with $20 \mathrm{mg}, 40 \mathrm{mg}$, or $60 \mathrm{mg}$ toremifene, or placebo, for 12 months, and 